\title{
Laparoscopic Evaluation of Female Factor in Bangladeshi Infertile Couple
}

\author{
UMME RUMAN ${ }^{1}$, TA CHOWDHURY², NUSRAT MAHMUD ${ }^{3}$, \\ TANJEEM S CHOWDHURY ${ }^{4}$, SAMIRA H HABIB ${ }^{5}$
}

\begin{abstract}
:
Background: Infertility prevails in good number in our society, causing social disharmony and distress. Laparoscopy is now a very effective tool for diagnosis and evaluation of female infertility.

Method: This cross sectional study was conducted in Infertility Unit of Bangladesh Institute of Research and Rehabilitation in Diabetes, Endocrine and Metabolic Disorders (BIRDEM) General Hospital, from July, 2012 to June 2014. The aim of the study is to identify common pelvic pathologies of female factor infertility by laparoscopy so that early detection and treatment modalities can be instituted.

Total 230 sub fertile patients attending OPD were selected for this study. Patients with DM, HTN, known case of hypothyroidism and male factor infertility was excluded from study. Detailed clinical history of every patient was recorded on a pre-set questionnaire and physical examination was performed. Laparoscopy was scheduled in proliferative phase of menstrual cycle.

Results: Among the study subjects, 130 (56.5\%) had primary infertility while 100 (43.5\%) secondary infertility. On laparoscopy, $64.4 \%$ patient had normal sized uterus, $31 \%$ had bulky uterus. Two patients had bicornuate uterus in secondary infertility cases. Uterus was restricted in mobility in $41 \%$ of patients due to endometriosis, pelvic inflammatory disease (PID) or previous pelvic surgery. Fibroid uterus was detected in 30(13\%) and 10(4.3\%) cases of primary and secondary infertility cases respectively. Bilateral tubal blockage was present in $16(6.9 \%)$ and $17(7.4 \%)$ cases of primary and secondary infertility respectively. Forty $(17.4 \%)$ cases of primary infertility were detected as polycystic ovaries (PCO) which was $30(13 \%)$ in cases of secondary infertility. Endometriosis was found in total $9(3.9 \%)$ patient in both the groups. Pelvic adhesion was found in 22(9.5\%) and 21 (9.1\%) cases of primary and secondary infertility respectively.
\end{abstract}

Conclusion: Laparoscopy could diagnose a substantial number of portion pelvic pathology. In a developing country like Bangladesh, earlier and justified intervention with laparoscopy can save time and expense of infertility treatment by diagnosing pelvic factor responsible for infertility.

Keywords: Laparoscopy, primary infertility, secondary infertility.

\section{Introduction:}

Infertility is defined as failure to conceive during one year of unprotected frequent intercourse ${ }^{1}$. It is a public health problem, both in developing and developed countries, affecting $10-15 \%$ of couples ${ }^{2}$. In many low-resource settings as in Bangladesh, infertility is common and frequently associated with negative psychosocial consequences including marital instability, divorce, social isolation and stigmatization ${ }^{3}$. In most of the cases ,treatment of infertility has a lengthy procedure and it is somehow expensive.

1. Classified Specialist, Obstetrics and Gynaecology, CMH, Dhaka. Bangladesh.

2. Professor, Department of gynae and obstetrics, BIRDEM General Hospital

3. Consultant, Centre for Assisted Reproduction, BIRDEM General Hospital

4. Assistant Professor, Department of Gynae and Obstetrics, BIRDEM General Hospital

5.Principal Research Officer, Health Economics Unit, Diabetic Association of Bangladesh

Address of Correspondence: Umme Ruman, Major, Classified Specialist, Obstetrics and Gynaecology, CMH, Dhaka. Bangladesh. Contact +8801711956262, E-mail: rumman09umme@yahoo.com 
Leading causes of infertility include tubal disease, ovulatory disorders, uterine or cervical factors, endometriosis and male factor. Major causes according to $\mathrm{WHO}$ on a global basis are malnutrition, pelvic tuberculosis and puerperal infections leading to tubal blockage ${ }^{4,5}$. Several studies describe risk factors for tubal pathology such as previous abdominal surgery and previous pelvic inflammatory disease (PID). However, up to $68 \%$ of patients without any of these risk factors can still possess abnormalities as shown by laparoscopy ${ }^{6-8}$.

Generally, laparoscopy is us usally reserved for couples who have already completed a more basic infertility evaluation including assessing for ovulation, ovarian reserve, ultrasound and hysterosalpingogram for the female and semen analysis for the male. Less invasive diagnostic tests such as patient's history, chlamydia antibody testing (CAT), ultrasonography and hysterosalpingography (HSG) are available, but it is still a matter of debate how the value of these tests compares with laparoscopy in the infertility work-up ${ }^{9}$. A meta-analysis of studies comparing Chlamydia antibody titres and laparoscopy for tubal pathology has demonstrated that discriminative capacity of chlamydial antibody titres in the diagnosis of tubal pathology is comparable to that of HSG. However it often fails to provide informations about severity of tubal disease which is important indicator to decide treatment options. It also is unable to detect endometriosis ${ }^{10}$. Early stages of endometriosis is a common factor for infertility which is easily detected by laparoscopy.

Bangladesh is an overpopulated developing country. Here, infertile couples are mostly treated by inexperienced hands initially. When they reach a tertiary centre where they are supposed to go earlier, it is already late. Laparoscopy helps us to detect pelvic pathology by direct visualization. Our present study is to see what are the common pelvic organ pathology affecting female partner in our country and whether early institution of laparoscopy benefit the patients.

\section{Methods and materials:}

All infertile couples attending in outpatient department of the Centre for Assisted Reproduction of BIRDEM General Hospital, Dhaka during the period of July, 2012 to June 2014 were enrolled. Patients suffering from both primary and secondary infertility were included in this study. Age of the patients were between 18 to 35 years. Patients who had medical disorders as DM, HTN, known case of hypothyroidism and male factor infertility was excluded from study.

Before admission, detailed clinical history was taken and clinical examination was done. A set of basic investigations were carried out for fitness of general anaesthesia including ultrasonography of lower abdomen before laparoscopy. Hysterosalpingography was not done routinely. If the patient had irregular menstruation, basic hormones (FSH, $\mathrm{LH}, \mathrm{TSH}$, Prolactin) were done to evaluate ovulatory defect. Finally patients selected for laparoscopy were done as an OPD procedure on 18-21 days of their menstrual cycle.

Data of the identified patients were collected from patient case records and it included factors such as age, duration and type of infertility, clinical examination findings, and gynecological ultrasound. Previous treatment history included details of ovulation stimulation, intrauterine insemination (IUI) and other treatment. Laparoscopy were performed in the morning. A laparoscope was inserted into the abdomen through the umbilicus. Carbon dioxide (CO2) gas was introduced into the abdomen prior to inserting the laparoscope. Uterus, Pouch of Douglus, fallopian tubes, ovaries were inspected for any pathology.

Intra operative findings such as presence of peritubal adhesions, endometriosis, tubal pathology, perihepatic adhesions, and hysteroscopic findings were recorded in a structured form. Dye test was done in each case. Minor interventions like ovarian drilling, adhesiolysis, removal of small cysts were performed in same sitting. Patients were discharged on the same day evening with necessary medications. Data analysis was done using SPSS 20. Informed consent was taken from every patient with proper explanation of risks and complications of the procedure. The study protocol was approved by ethical committee of BIRDEM hospital.

\section{Results:}

Two thirty patients were included for diagnostic laparoscopy out of total 550 infertile patients. In this series, 130 (56.5\%) patients were in primary infertility group and $100(43.5 \%)$ patients were in secondary infertility group. Most of the patients $(59.6 \%)$ ages were between 20-30 years (Fig-1). Duration of infertility was $2-5$ years in $46 \%$ patients whereas $30 \%$ patients had been infertile for $5-10$ years.57.8\% patients had regular menstrual cycle.

Patients with secondary infertility reported $10 \%$ with alive issues, $1 \%$ still birth, $29 \%$ had history of abortion, $13.5 \%$ had history of menstrual regulation and $3 \%$ had previous ectopic pregnancy. Total II patients had history of appendicitis, 5 patients had tuberculosis and 18 patients underwent some kind of pelvic surgery. 
On laparoscopy, $64.4 \%$ patients had uterus of normal size; $40 \%$ patient had irregular shaped uterus due to fibroid most of which belongs to primary infertility group. One hundred out of 130 primary infertility patients had regular shaped uterus (Table-I). Almost

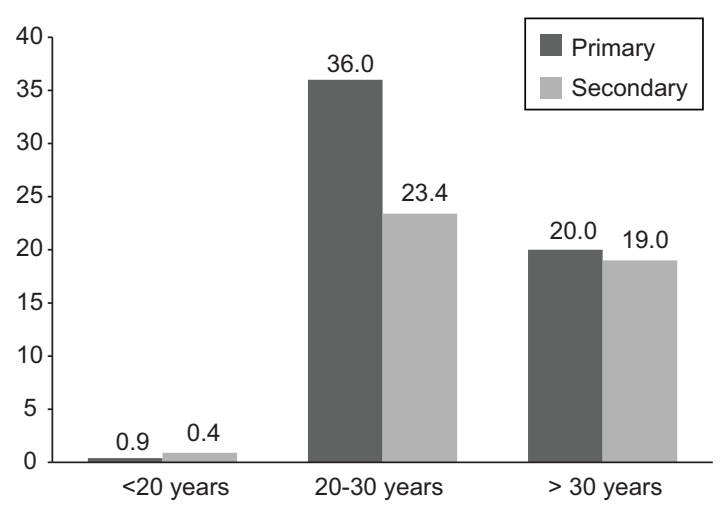

Fig.-1: Distribution of subjects according to their age $(n=230)$

equal number of patients (20 and 21) of both group were found to have pelvic adhesion. Endometriotic lesions were found in pouch of Douglus in $4 \%$ patients. Pouch of Douglus was free in $77.4 \%$ patients (Fig-2).

Normal fallopian tube was present on right side in $70 \%$ and on left side in $84.3 \%$ of patients. We found peritubal adhesion more in right tube than left tube (22\% in right tube and $14 \%$ in left tube). Number of hydrosalpynx is almost equal in both groups. Bilateral tubal block was present in 33(14.3\%) patients. Single tubal block in either right or left side was found in 22 and $25 \%$ of cases respectively (Fig-3). Detailed findings of both fallopian tubes are shown in Table- II.

In Table-III, no ovarian pathology was found in 77-79 $\%$ of patients. We see that polycystic ovary was found higher number in primary infertility group $(17.4 \%)$ than that of secondary infertility (13\%). Chocolate cyst was also more common in primary infertility patients. More detailed comparison is given in Table -III.

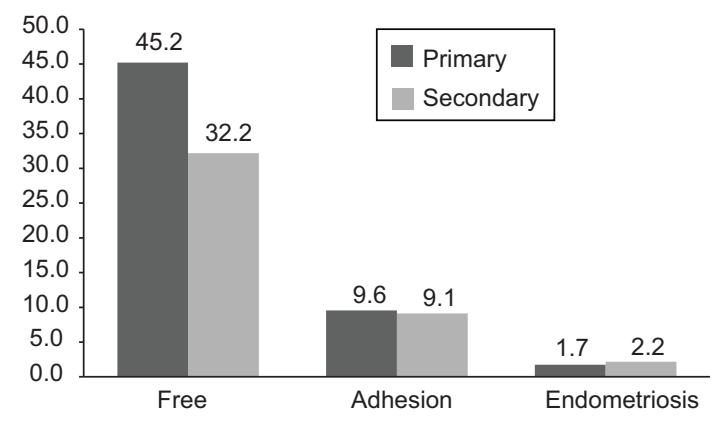

Fig 2: Distribution of subjects according to

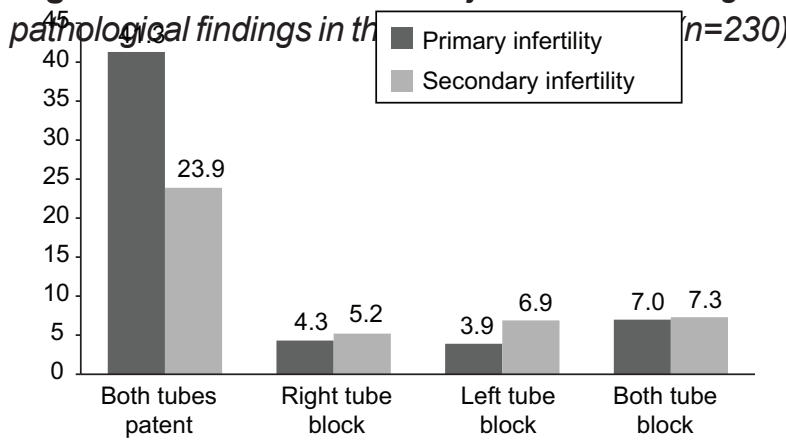

Fig.-3: Distribution of study subjects according to tubal patency test $(n=230)$

Table-I

Distribution of study subjects according to laparoscopic findings of uterine size, shape, mobility ( $n=230)$

\begin{tabular}{lccc}
\hline $\begin{array}{l}\text { Laparoscopic } \\
\text { findings }\end{array}$ & $\begin{array}{c}\text { Primary infertility } \\
\text { Number(\%) }\end{array}$ & $\begin{array}{c}\text { Secondary infertility } \\
\text { Number(\%) }\end{array}$ & Total \\
\hline Uterine size & & & \\
Normal & $88(38.2)$ & $60(26)$ & $148(64.4)$ \\
Smaller & $7.0(3)$ & $3.0(1.3)$ & $10(4.3)$ \\
Bulky & $35(15)$ & $37(16)$ & $72(31.3)$ \\
Uterine shape & & & \\
Regular & $100(43.5)$ & $88(38.3)$ & $196(85.1)$ \\
Irregular & $30(13))$ & $10(4.3)$ & $40(17.3)$ \\
Bicornuate & - & $2(0.9)$ & $2(0.9)$ \\
Mobility & $109(47)$ & $80(34.8)$ & $189(82.2)$ \\
Mobile & $21(9.13)$ & $20(8.7)$ & $41(17.8)$ \\
Restricted & & & \\
\hline
\end{tabular}


Table-II

Distribution of study subjects according to laparoscopic findings of fallopian tubes $(n=230)$

\begin{tabular}{lccc}
\hline $\begin{array}{l}\text { Laparoscopic } \\
\text { findings }\end{array}$ & $\begin{array}{c}\text { Primary infertility } \\
\text { Number(\%) }\end{array}$ & $\begin{array}{c}\text { Secondary infertility } \\
\text { Number(\%) }\end{array}$ & Total \\
\hline Right tube & $114(49.6)$ & & $160(69.6)$ \\
Normal & $10(4.34)$ & $46(20)$ & $51(22.1)$ \\
Adhesion & $2(0.9)$ & $41(17.8)$ & $7(3.04)$ \\
Kinked & - & $5(2.2)$ & $3(1.3)$ \\
Absent & $4(1.7)$ & $3(1.3)$ & $9(3.9)$ \\
Hydrosalpynx & & $5(2.2)$ & \\
Left tube & $115(50)$ & & $194(84.3)$ \\
Normal & $7(6.5)$ & $79(34.3)$ & $14(6.0)$ \\
Adhesion & $1(0.43)$ & $7(3.04)$ & $4(1.7)$ \\
Kinked & - & $3(1.3)$ & $3(1.3)$ \\
Absent & $7(3.04)$ & $3(1.3)$ & $15(6.5)$ \\
Hydrosalpynx & & $8(3.4)$ & \\
\hline
\end{tabular}

Table-III

Distribution of study subjects according to the laparoscopic findings of ovaries $(n=230)$

\begin{tabular}{lccc}
\hline $\begin{array}{l}\text { Laparoscopic } \\
\text { findings }\end{array}$ & $\begin{array}{c}\text { Primary infertility } \\
\text { Number(\%) }\end{array}$ & $\begin{array}{c}\text { Secondary infertility } \\
\text { Number(\%) }\end{array}$ & Total \\
\hline Right ovary & & & \\
Normal & $44(19.1)$ & $35(15.2)$ & $79(34.3)$ \\
Adhesion & $10(4.3)$ & $11(4.8)$ & $21(9.1)$ \\
Smaller & $4(1.70$ & $1(0.43)$ & $5(2.1)$ \\
Multicystic & $13(5.7)$ & $18(7.8)$ & $31(13.4)$ \\
Polycystic & $40(17.4)$ & $30(13)$ & $70(30.4)$ \\
Chocolate cyst & $19(18.3)$ & $5(2.2)$ & $24(10.4)$ \\
Left ovary & & & \\
Normal & $48(20.9)$ & $29(12.7)$ & $77(33.4)$ \\
Adhesion & $8(3.5)$ & $13(5.7)$ & $21(9.1)$ \\
Smaller & $4(1.7)$ & $1(0.4)$ & $5(2.1)$ \\
Multicystic & $18(7.8)$ & $22(9.6)$ & $38(16.5)$ \\
Polycystic & $40(17.4)$ & $30(13)$ & $72(31.3)$ \\
Chocolate cyst & $12(5.2)$ & $5(2.2)$ & $17(7.4)$ \\
\hline
\end{tabular}

\section{Discussion:}

Introduction of laparoscopy has tremendously improved the ability to investigate long standing infertility. Uterus, fallopian tubes, ovaries can be directly visualized by laparoscopy and information about present pelvic pathology can be obtained.

In this study, we have found 66.7 women $(29 \%)$ had history of abortion and 27 women (13.5\%) had history of menstrual regulation(MR). A similar study was performed by Biswas $R$ et al where secondary infertility following post abortion was $18 \%$, following
MR was $21.5 \%$ and following still birth $9 \%{ }^{11}$. Here tubal pathology might have occured following gonococcal, chlamydial, or other STD causing asymptomatic pelvic inflammatory disease (PID). Another study done in sub-Saharan countries showed tubal factors (such as unilateral or bilateral tubal occlusion, pelvic adhesion, and other abnormalities) in $32 \%$. About $66 \%$ of African women experienced tubal factors compared to about $33 \%$ worldwide. About $9 \%$ of women reported a history of sexually transmitted disease (STD), and $8 \%$ reported abortion complications $^{12}$. 
In our study, 18(7.8\%) patient had pelvic surgery, $11(4.8 \%)$ had history of appendicitis, $5(2 \%)$ had history of pulmonary tuberculosis. More of these patients belonged to secondary infertility group. Hovav $Y$ et al studied the role of diagnostic laparoscopy in primary and secondary infertility. In only 3 of the 20 patients with primary infertility was there no history of an pelvic surgery, pelvic inflammatory disease, or an abnormal hysterosalpingography of the 62 women who suffered secondary infertility and had positive findings in laparoscopy, 15 had no history of suspect findings ${ }^{13}$.

In our study, on laparoscopy, $30(13 \%)$ patients of primary infertility group had irregular uterus due to fibroid. There was adhesion in Pouch of Douglus in $9.6 \%$ of patients with primary infertility which was a bit higher than secondary infertility. Pelvic endometriosis was found in $2.2 \%$ of patients of secondary infertility. In a case series published by Aziz $\mathrm{N}$ et al , endometriosis was found in 4 (12.5\%) cases with primary infertility and $2(11.1 \%)$ cases with secondary infertility. Pelvic inflammatory disease (PID) was found in $1(3.1 \%)$ and $2(16.7 \%)$ cases of primary and secondary infertility respectively. Bicornuate uterus was present in 1 cases of secondary infertility ${ }^{14}$. In a study of 100 infertile petients, Biswas $\mathrm{R}$ et al showed $9 \%$ had fibroid uterus, $2 \%$ had bicornuate uterus and $18 \%$ had bulky uterus $^{11}$. We found only 2 (9\%) patients with bicornuate uterus in our study.

In the present study, in cases of secondary infertility both tubes were blocked in $17(7.4 \%)$ cases and both tubes were patent in 55(23.9\%) cases and only one tube was blocked in 28 (20.5\%) cases. A similar study was done by Chowdhury $S$ et al where tubal blockage was found in $15 \%$ cases of primary infertility and $17.5 \%$ cases of secondary infertility ${ }^{15}$. This finding is much lower than the series by Collet Mwhere the study was done in Eastern Gabon in the infertility belt, where the tubal occlusion was present in $82.2 \%$ cases. This high prevalence of tubal occlusion in Africa was probably due to sexual promiscuity, which play a major role in causing secondary subfertility ${ }^{16}$. Bilateral tubal occlusion was found in $35.5 \%$ cases in laparoscopy in infertile patients of Nigeria shown in a study by OtolorinEO et al which is higher than our study. The higher prevalence might be due higher incidence of STD in Nigeria because of freedom of sexual activity in the society ${ }^{17}$.
It is known that incidence of tubal factors in case of primary infertility is about $15 \%$ and in secondary infertility $40 \%{ }^{18}$. Different types of tubal pathology were observed laparoscopically such as peritubal adhesion, hydrosalpynx, agglutination of fimbria, tubal blockage (unilateral/ bilateral) which played important role in both primary and secondary infertility. A study by Gulfareen $\mathrm{H}$ et al has shown that the commonest finding by laparoscopy in patients with primary infertility was endometriosis which accounted for $11(55 \%)$ while in secondary infertility tubal occlusion was more common which accounted for $3(30 \%)$ cases $^{19}$.

In a cross sectional study published by Naz et al, ovarian pathology was found in $18(13.23 \%)$ cases of primary and $4(7.01 \%)$ cases of secondary infertility. Polycystic ovaries (PCO) were detected in 12 (8.82\%) and $2(3.5 \%)$ cases of primary and secondary infertility respectively. Endometriotic cysts and deposits were found in $15(10.29 \%)$ cases of primary and $3(5.26 \%)$ cases of secondary infertility[4]. In our study, findings show \& periovarian adhesion in $4.3 \%$ cases of primary and $4.8 \%$ in cases of secondary infertility which is significant in secondary infertility. Frequency of PCOS and chocolate cyst was higher in primary infertility. PCOS was present in $17.4 \%$ patients of primary infertility and $13 \%$ patients of secondary infertility. Chocolate cyst was present in $23.5 \%$ patients with primary and $4.4 \%$ of secondary infertility. A study by Aziz N showed five (15.6\%) cases of primary infertility were detected as polycystic ovaries (PCO) which was not found in cases of secondary infertility. Endometriosis was found in $4(12.5 \%)$ cases with primary infertility and $2(11.1 \%)$ cases with secondary infertility[14]. A similar study by Khan Ml in Bangladesh in a study of 50 infertile patients, has shown pelvic pathology like endometriosis was present in $5(10 \%)$ cases ,adhesion was detected in $14(28 \%)$ and chocolate cyst was found in $3(6 \%)$ cases[20]. In another similar study, Shetty SK et al showed endometriosis in $12(24 \%)$ cases and peritubal adhesion in $4(8 \%)$ cases where endometriosis was higher but adhesion was lower than our study[21]. A prospective study conducted by Boricha YG et al showed $10(20 \%)$ had tubal pathology out of $50,7(14 \%)$ cases had unilateral block and in $3(6 \%)$ cases both tubes were blocked ${ }^{22}$.

We could conclude that common causes responsible for infertility in this study were tubal occlusion, 
endometriosis, peritubal and periovarian adhesions. Ovarian causes were seen in primary infertility predominantly. On the other hand, tubo-peritoneal factors were predominantly present in cases of secondary infertility.

In a poor country like Bangladesh, most of the patients initially go to untrained health practitioners for treatment of infertility leads to further delay in proper management. Laparoscopy not only helps in identification of unsuspected pathology but also contributes to decision making. So it should be considered as an initial and important investigations for the evaluation of infertile couple.

\section{Conclusion:}

From this study, it can be concluded that tubal blockage, pelvic adhesion and endometriosis are important tuboperitoneal factors in infertility. Laparoscopic procedures are more convenient and more precise for diagnosis of pelvic pathology. There should be proper guidance and education of infertile women to consult earlier at proper infertility clinic. Early decision for laparoscopy specially in case of secondary infertility can be cost effective and minimize time for decision making. There is need to establish the role of laparoscopy in both the diagnosis and management of patients with infertility and larger studies need to be carried out.

\section{References:}

1. Jose-Miller, A.B., J.W. Boyden, and K.A. Frey, Infertility. Am Fam Physician, 2007. 75(6): p. 849-56.

2. Templeton, A., Infertility-epidemiology, aetiology and effective management. Health bulletin, 1995. 53(5): p. 294-298.

3. Van Balen, F. and H. Bos, The social and cultural consequences of being childless in poorresource areas. F, V\&V in ObGyn, 2009. 1(2): p. 106-21.

4. Naz, T., et al., Laparoscopic evaluation in infertility. J Coll Physicians Surg Pak, 2009. 19(11): p. 704-7.

5. Mehmood, S., An audit of diagnostic laparoscopies for infertility. J Surg Pak, 2003. 8(3): p. 8-10.

6. Donnez, J., et al., Incidence of pathological factors not revealed by hysterosalpingography but disclosed by laparoscopy in 500 infertile women. European Journal of Obstetrics \& Gynecology and Reproductive Biology, 1982. 13(6): p. 369-375.

7. Musich, J.R. and S.J. Behrman, Infertility laparoscopy in perspective: review of five hundred cases. American journal of obstetrics and gynecology, 1982. 143(3): p. 293-303.

8. Corson, S.L., A. Cheng, and N.G. Jacqueline, Laparoscopy in the "normal" infertile patient: a question revisited. The Journal of the American Association of Gynecologic Laparoscopists, 2000. 7(3): p. 317-324.

9. Tanahatoe, S., P.G. Hompes, and C.B. Lambalk, Accuracy of diagnostic laparoscopy in the infertility work-up before intrauterine insemination. Fertility and sterility, 2003. 79(2): p. 361-366.

10. Mol, B.W., et al., The accuracy of serum chlamydial antibodies in the diagnosis of tubal pathology: a meta-analysis. Fertility and sterility, 1997. 67(6): p. 1031-1037.

11. Biswas, R. and T. Chowdhury, Laparoscopic evaluation of tubal pathology in cases of infertility. Ibrahim Medical College Journal, 2014. 7(2): p. 38-40.

12. Gerais, A. and H. Rushwan, Infertility in Africa. Population sciences (Cairo, Egypt), 1992. 12: p. 25.

13. Hovav, Y., et al., Diagnostic laparoscopy in primary and secondary infertility. Journal of assisted reproduction and genetics, 1998. 15(9): p. 535-537.

14. Aziz, N., Laparoscopic evaluation of female factors in infertility. J Coll Physicians Surg Pak, 2010. 20(10): p. 649-52.

15. Chowdhury S, C.T., Laparoscopic assessment of tubal factors in infertility. Bangladesh Journal of Obstetrics and Gynaecology, 1992. 7(1): p. 9-16.

16. Collet, M., et al., Infertility in Central Africa: infection is the cause. International Journal of Gynecology \& Obstetrics, 1988. 26(3): p. 423-428. 
17. Otolorin, E.O., O. Ojengbede, and A. Falase, Laparoscopic evaluation of the tuboperitoneal factor in infertile Nigerian women. International Journal of Gynecology \& Obstetrics, 1987. 25(1): p. 47-52.

18. Edmonds, D., Infertility, Dewhart's textbook of Obstetrics and Gynaecology for postgraduates. 8 ed. 2012, London: Blackwell Science Ltd.

19. Haider, G., et al., Laparoscopic evaluation of female infertility. J Ayub Med Coll Abbottabad, 2010. 22(1): p. 136-38.
20. Khan, M., et al., Laparoscopic Evaluation of the Tuboperitoneal Factors in Infertility. BIRDEM Medical Journal, 2014. 4(1): p. 9-12.

21. Shetty, S.K., H. Shetty, and S. Rai, Laparoscopic evaluation of tubal factor in cases of infertility. International Journal of Reproduction, Contraception, Obstetrics and Gynecology, 2013. 2(3): p. 410-413.

22. Boricha YG, S.R., Boricha BG, Mhapankar S, Chatterjee A, Nashetty J, Laparoscopy in 50 infertile patients: A prospective study. International journal of Medical and Clinical Research, 2011. 2(2): p. 63-66. 